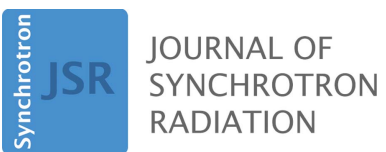

ISSN 1600-5775

Received 25 June 2019

Accepted 4 November 2019

Edited by A. Stevenson, Australian Synchrotron, Australia

Keywords: resonant inelastic X-ray scattering; $\mathrm{X}$-ray photoelectron spectroscopy; soft X-ray spectroscopy; soft X-ray beamline;

$\mathrm{X}$-ray emission; X-ray absorption; BESSY II.

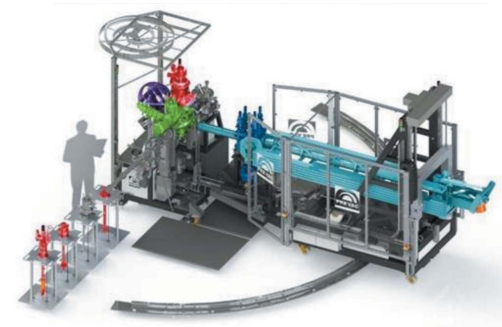

OPEN ๑ ACCESS

\section{Characterization of the soft X-ray spectrometer PEAXIS at BESSY II}

\author{
Christian Schulz, ${ }^{a}$ Klaus Lieutenant, ${ }^{\text {a }}$ Jie Xiao, ${ }^{\mathrm{b}}$ Tommy Hofmann, ${ }^{\mathrm{a}}$ Deniz Wong ${ }^{\mathrm{a}}$ \\ and Klaus Habicht ${ }^{\mathrm{a}, \mathrm{c} *}$
}

\begin{abstract}
a Department of Methods for Characterization of Transport Phenomena in Energy Materials, Helmholtz-Zentrum Berlin für Materialien und Energie, D-14109 Berlin, Germany, 'bepartment of Highly Sensitive X-ray Spectroscopy, HelmholtzZentrum Berlin für Materialien und Energie, D-14109 Berlin, Germany, and 'Institut für Physik und Astronomie, Universität Potsdam, D-14476 Potsdam, Germany. *Correspondence e-mail: habicht@helmholtz-berlin.de
\end{abstract}

The performance of the recently commissioned spectrometer PEAXIS for resonant inelastic soft X-ray scattering (RIXS) and X-ray photoelectron spectroscopy and its hosting beamline U41-PEAXIS at the BESSY II synchrotron are characterized. The beamline provides linearly polarized light from $180 \mathrm{eV}$ to $1600 \mathrm{eV}$ allowing for RIXS measurements in the range 200$1200 \mathrm{eV}$. The monochromator optics can be operated in different configurations to provide either high flux with up to $10^{12}$ photons s ${ }^{-1}$ within the focal spot at the sample or high energy resolution with a full width at half maximum of $<40 \mathrm{meV}$ at an incident photon energy of $\sim 400 \mathrm{eV}$. The measured total energy resolution of the RIXS spectrometer is in very good agreement with theoretically predicted values obtained by ray-tracing simulations. PEAXIS features a $5 \mathrm{~m}$-long RIXS spectrometer arm that can be continuously rotated about the sample position by $106^{\circ}$ within the horizontal photon scattering plane, thus enabling the study of momentum-transfer-dependent excitations. Selected scientific examples are presented to demonstrate the instrument capabilities, including measurements of excitations in single-crystalline $\mathrm{NiO}$ and in liquid acetone employing a fluid cell sample manipulator. Planned upgrades of the beamline and the RIXS spectrometer to further increase the energy resolution to $\sim 100 \mathrm{meV}$ at $1000 \mathrm{eV}$ incident photon energy are discussed.

\section{Introduction}

The electronic structure and dynamics determine important fundamental properties of materials such as charge and spin transport, novel phases of matter and emergent phenomena originating in strong electronic correlations. Thus probing electronic excitations and the coupling of electrons to lattice, orbital and spin degrees of freedom provides an invaluable tool to understand the microscopic mechanisms which determine macroscopic functionalities for technological applications.

Resonant inelastic X-ray scattering (RIXS) at brilliant third-generation synchrotron radiation sources nowadays is a mature technique known to access element-specific information on the electronic structure and dynamics as well as to gain momentum-resolved information on quasiparticle excitations which couple to the electronic states. Being a photon-in/ photon-out spectroscopic technique, RIXS is bulk-sensitive and thus well suited to investigate ordered solid state materials. In the soft X-ray regime, RIXS allows for the study of resonant excitations of electronic states in important elements such as $\mathrm{C}, \mathrm{N}, \mathrm{O}$ and the series of $3 d$ transition metal elements present in a broad range of materials of fundamental and technological interest. 
The technique has recently been applied to address important scientific questions in quantum materials with breakthrough results identifying charge collective modes as acoustic plasmons in electron-doped copper oxide superconductors (Hepting et al., 2018), probing multi-spinon excitations in spin 1/2 one-dimensional Heisenberg antiferromagnets (Schlappa et al., 2018) or extracting the electron-phonon coupling strength in oxide heterostructures (Meyers et al., 2018). Beyond numerous applications for RIXS in solid state physics, intra- and inter-molecular couplings can be studied in molecular systems either in the liquid phase (Vaz da Cruz et al., 2019) or in the gas phase (Couto et al., 2017).

Numerous soft X-ray RIXS spectrometers have been built at synchrotron facilities in the recent past, such as SAXES at SLS, PSI (Strocov et al., 2010), SEXTANTS at SOLEIL (Chiuzbăian et al., 2014), RIXS at BL05A at the TLS (Huang \& Chen, 2018), iRIXS at ALS (Chuang et al., 2017), SIXS at NSLS II (Jarrige et al., 2018), ID32 at ESRF (Brookes et al., 2018) and $\mathrm{I} 21$ at Diamond Light Source (http://www. diamond.ac.uk/Beamlines/Spectroscopy/I21.html), or are currently either in commissioning or in the planning stage, such as VERITAS at MAX IV (https://www.maxiv.lu.se/ accelerators-beamlines/beamlines/veritas/) and METRIX at BESSY II (Pietzsch et al., 2018), to address the increasing demand for RIXS capabilities. The new spectrometer PEAXIS (Photo Electron Analysis and resonant X-ray Inelastic Spectroscopy) at the third-generation synchrotron BESSY II adds to the capacity of RIXS spectroscopy for the international user community. It has been built with a particular focus on solid state applications and allows for spectroscopy of liquids encapsulated in sealed cells. In addition, X-ray photoelectron spectroscopy (XPS) capabilities are offered. In this article, important instrumental parameters of the hosting beamline and the spectrometer are presented and the overall performance of PEAXIS is demonstrated on selected experimental examples.

\section{Beamline layout and performance}

\subsection{Beamline design}

PEAXIS is a fixed station installed at undulator U41 of BESSY II which has $41 \mathrm{~mm}$ period length and minimum gap of $15.5 \mathrm{~mm}$ and delivers linearly polarized light with horizontal polarization. The optical layout of the beamline is shown in Fig. 1. It is based on a plane-grating monochromator (PGM) design, which can achieve high energy resolution, high flux and a wide energy range coverage by employing a single grating only. A first toroidal mirror $\left(\mathrm{M}_{1}\right)$ collimates the divergent beam from the undulator source in the horizontal plane and provides a parallel beam in the vertical plane. The next optical element is the monochromator which disperses the beam according to its photon energies. It consists of a plane pre-mirror $\left(\mathrm{M}_{2}\right)$ and a plane blazed grating $(\mathrm{G})$ assembly in which the grating has a groove density of 800 lines $\mathrm{mm}^{-1}$. The monochromator can be aligned to different fixed-focus constants $c_{\mathrm{ff}}=\cos \beta / \cos \alpha$. Here $\alpha$ and $\beta$ are the incident and exit angles at the monochromator grating (cf. Section 3.2). A second toroidal mirror $\left(\mathrm{M}_{3}\right)$ then focuses the spatially extended beam in both the vertical and the horizontal planes onto the exit slit, providing monochromatic light after the slit. Finally, an ellipsoidal mirror $\left(\mathrm{M}_{4}\right)$ refocuses the beam onto the sample position in the PEAXIS sample chamber.

\subsection{Photon flux, focal spot size and energy resolution}

By combining different $c_{\mathrm{ff}}$ values with appropriate exit slit widths, the beamline can be tuned for either higher energy resolution or increased photon flux. The three typically chosen modes of operation are a high-flux mode $\left(c_{\mathrm{ff}}=2.25\right.$, slit size $20 \mu \mathrm{m})$, a standard mode $\left(c_{\mathrm{ff}}=3\right.$, slit size $\left.10 \mu \mathrm{m}\right)$ and a highresolution mode $\left(c_{\mathrm{ff}}=5\right.$, slit size $\left.5 \mu \mathrm{m}\right)$, though intermediate combinations of $c_{\mathrm{ff}}$ and slit size can be freely chosen to match the experimental requirements. Changing both $c_{\mathrm{ff}}$ and the exit slit gap rather than changing the exit slit gap while keeping $c_{\mathrm{ff}}$ fixed results in improved flux for a given energy resolution. For the three typical settings, the incident photon flux at the focal point of the beamline at the sample position within the PEAXIS chamber has been determined by total electron yield measurements on a clean Au sample and by recording the current on a windowless GaAsP photodiode (Hamamatsu G1127-04). These measurements were carried out for different 


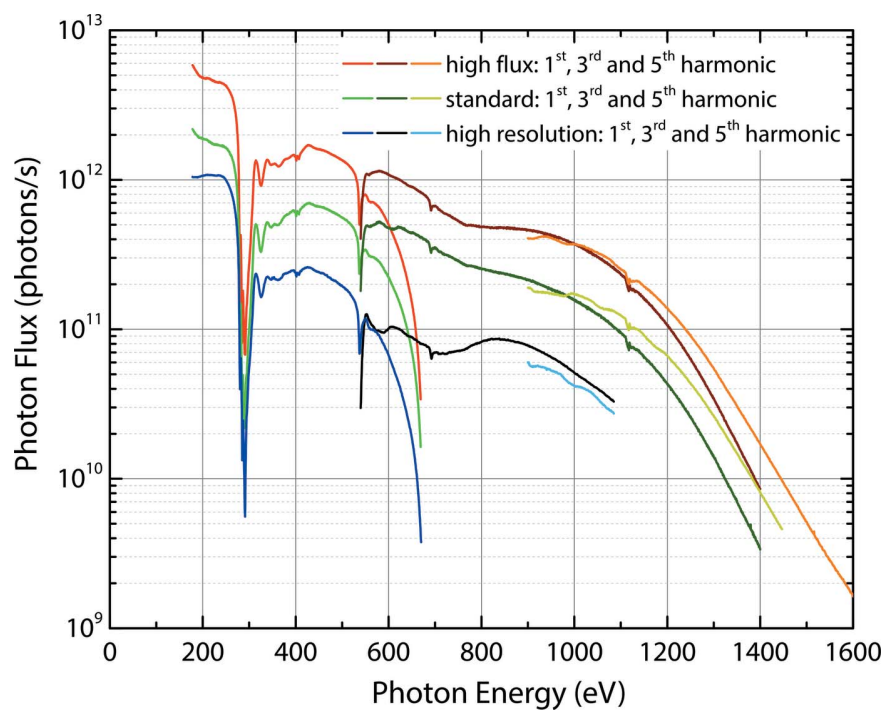

Figure 2

X-ray photon flux at the sample position for the three operation modes of the beamline and first, third and fifth harmonics from the undulator. The measurements were taken under normal multi-bunch operation of the BESSY II storage ring with a current of $250 \mathrm{~mA}$ and an opening of the beamline entrance slits of $2 \mathrm{~mm} \times 3 \mathrm{~mm}$ (standard use).

undulator harmonics covering the whole energy range of the beamline.

The results of these flux measurements are shown in Fig. 2 for the three typical modes of operation. Note that for the high-resolution mode $\left(c_{\mathrm{ff}}=5\right.$ and slit opening $\left.5 \mu \mathrm{m}\right)$ the photon energy of the beamline is limited to $1085 \mathrm{eV}$, as a significant fraction of the photon beam will not be reflected by the pre-mirror $\left(\mathrm{M}_{2}\right.$ in Fig. 1) in front of the grating for higher incident photon energies.

The focal spot size was determined by measuring the induced electric current on a gold-coated knife-edge moved incrementally into the beam until reaching current saturation for full coverage of the focal spot in both vertical and horizontal directions. The resulting step functions were fitted to obtain the full width at half maximum (FWHM) of the assumed Gaussian spot profiles [shown for the vertical spot size in Fig. 3(a)]. We note that our fit model relies on the integration of a single Gaussian and neglects both (i) an enhanced electron emission close to the edge of the knife-edge and (ii) a possible additional broadening beyond a single Gaussian to describe the vertical spatial beam profile. While the former effect leads to the decrease of the measured current above $17.135 \mathrm{~mm}$ [Fig. 3(a)], the latter effect partially accounts for the increased current in the interval $17.215 \mathrm{~mm}$ to $17.130 \mathrm{~mm}$. However, this does not significantly change the resulting FWHM which we use here to demonstrate the performance of the beamline optics. For the high-flux mode of operation, we obtain a maximum focal spot size of $3.8 \mu \mathrm{m} \times$ $12.4 \mu \mathrm{m}$ FWHM (vertical $\times$ horizontal). This small vertical spot size is important for a good energy resolution of the RIXS spectrometer, because it defines the deviation from the optimal incident angle $\alpha$ to the grating in the RIXS spectrometer.
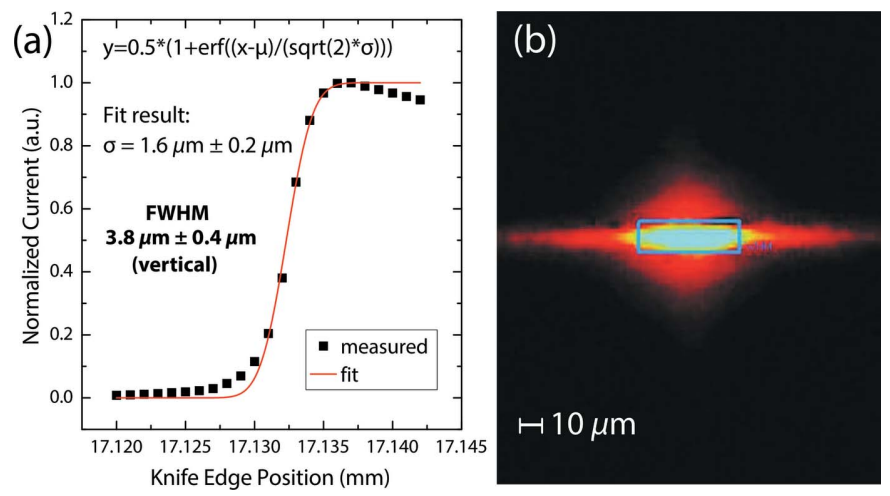

Figure 3

(a) Fit of knife-edge measurement data to determine the (vertical) Gaussian FWHM of the focal spot size. (b) Two-dimensional image of the focal spot of the beamline at the sample position, recorded by a CCD with high spatial resolution in the high-flux mode of operation of the beamline.

Additionally, a two-dimensional image of the focal spot was recorded by using a dedicated vacuum chamber placed at the sample position prior to the installation of the PEAXIS sample chamber. This measurement setup allows positioning of a scintillator detector which converts the X-ray image into visible light at the focal spot of the beamline. The image is subsequently magnified by an optical zoom lens and detected by a CCD camera, both being located outside the vacuum chamber. Compared with the RIXS setup, for example, the lens in this setup can be positioned very close to the scintillator to obtain a numerical aperture $n \sin \phi$, with refractive index $n$ and aperture angle $\phi$, which provides the required high spatial resolution (Martin \& Koch, 2006). Nevertheless, there is a discrepancy between the focal spot size determined by this method compared with that determined from the knifeedge measurements which we attribute to inherent scattering of the visible light within the scintillator and the glass window behind it.

The resolving power of the beamline was determined from absorption measurements in $\mathrm{N}_{2}$ and $\mathrm{Ne}$ gas, the latter of which is also used for the calibration of the monochromator setup for an absolute value of the incident photon energy. These two gases have resonance energies of $\sim 400 \mathrm{eV}$ and $\sim 870 \mathrm{eV}$, respectively, which allows the beamline performance to be characterized at two pertinent incident photon energy regions in the PEAXIS energy range of operation.

For the lower-energy region, the photon absorption of $\mathrm{N}_{2}$ at the $1 s \rightarrow \pi^{*}$ resonance around $401 \mathrm{eV}$ leads to a fine structure due to vibrational levels of the $\pi^{*}$ state, whose peak positions and linewidths are routinely used to determine the intrinsic instrumental resolution of soft X-ray beamlines (Feifel et al., 2004). The recorded photoabsorption spectrum of $\mathrm{N}_{2}$ for a gas cell pressure of $2 \times 10^{-3} \mathrm{hPa}$ and the high-resolution mode of the PEAXIS beamline is shown in Fig. 4(a). The spectrum is characterized by the appearance of eight vibrational levels which are fitted each with a convolution of a Gaussian lineshape representing the intrinsic energy resolution of the beamline (in FWHM) and a Lorentzian lineshape to represent the $\mathrm{N} 1 s$ core-hole lifetime broadening. Measurements with 
Table 1

Energy resolution, resolving power and photon flux of the beamline for the high flux, standard and high-resolution modes of operation, measured on $\mathrm{N}_{2}$ and $\mathrm{Ne}$ gas absorption lines for energy regions around $401 \mathrm{eV}$ and $867 \mathrm{eV}$, respectively.

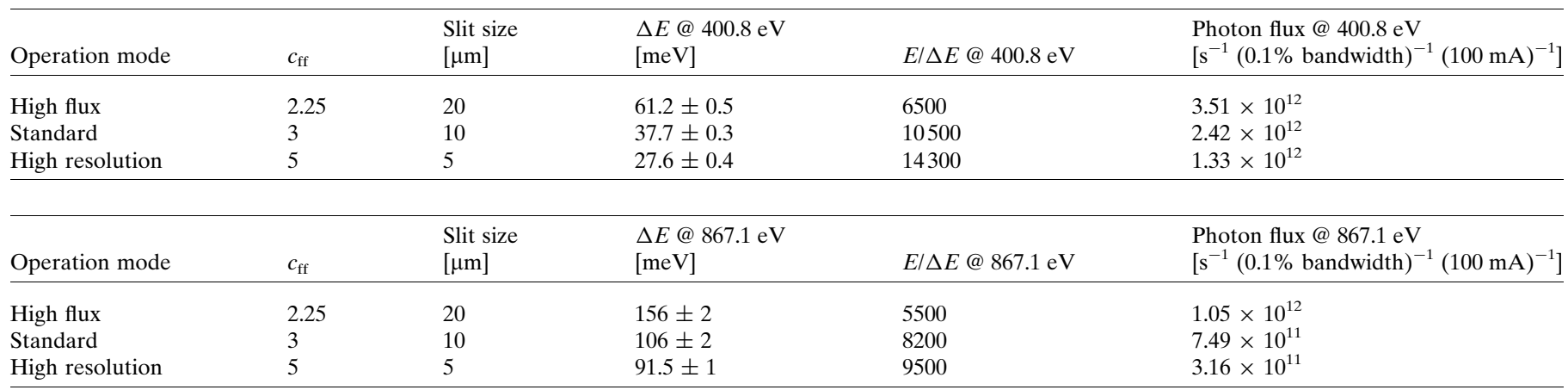

different settings for $c_{\mathrm{ff}}$, slit size and diffraction order of the undulator have been used to determine the Lorentzian width to a mean value of $120 \pm 2 \mathrm{meV}$ FWHM, which is in good agreement with values found in the literature (Feifel et al., 2004; Kato et al., 2007a). Based on this value for the core-hole lifetime broadening in $\mathrm{N}_{2}$, the Gaussian FWHM of the beamline was found to be $27.6 \pm 0.4 \mathrm{meV}$. For the incident energy of $400.8 \mathrm{eV}$, this energy resolution of $\sim 28 \mathrm{meV}$ thus corresponds to a resolving power $E / \Delta E$ exceeding 14300 , which is close to the resolution limit of this method due to the uncertainty of the Lorentzian broadening. To circumvent this limitation, the combined energy resolution of the beamline and the RIXS spectrometer are evaluated by linewidth
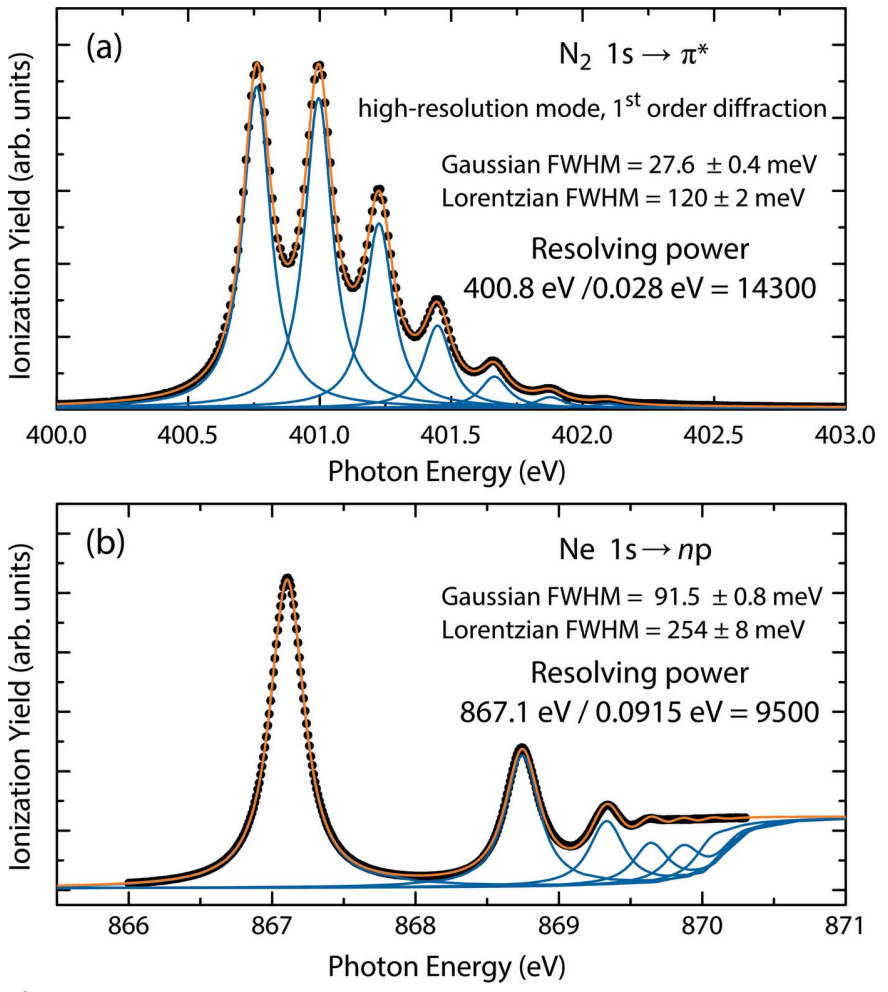

Figure 4

Beamline energy resolution as measured from $(a) \mathrm{N}_{2}$ and $(b) \mathrm{Ne}$ gas photoabsorption. Both spectra were measured in first diffraction order and in the high-resolution mode of the beamline. For details, see text. measurements of the elastically scattered X-rays on a polished NiO crystal surface (see Section 3.2).

At higher incident photon energy, the energy resolution of the beamline was determined from the $1 s^{-1} \rightarrow n p$ Rydberg series of Ne under the same experimental conditions as for the $\mathrm{N}_{2}$ measurements discussed above. Here, a Lorentzian FWHM of $254 \pm 8 \mathrm{meV}$ was obtained by fitting the $1 s^{-1} \rightarrow 3 p$ peak for different beamline settings. This Lorentzian width is in good agreement with values found in the literature (Kato et al., 2007b). The Ne photoabsorption spectrum shown in Fig. 4(b) was then fitted with a series of Voigt profiles for the $1 s^{-1} \rightarrow n p$ ( $n=3-8$ ) Rydberg series on top of a nonlinear background at high energies related to the $\mathrm{Ne} 1 s$ core ionization threshold at $870.16 \mathrm{eV}$. A common Gaussian FWHM of $91.5 \pm 0.8 \mathrm{meV}$ was so determined as the intrinsic energy resolution at the incident energy of $867.1 \mathrm{eV}$, corresponding to a resolving power of 9500 for the high-resolution mode of operation. The obtained resolution parameters for the typical operation modes of the beamline from $\mathrm{N}_{2}$ and $\mathrm{Ne}$ measurements are listed in Table 1.

\section{PEAXIS instrument and characterization}

PEAXIS combines two important types of measurement techniques, namely wavevector-dependent RIXS and XPS, in a single instrument. The RIXS instrument is based on an inhouse design concept previously discussed (Lieutenant et al., 2016a), while the XPS analyzer is a commercial 'PHOIBOS 150 EP' hemispherical analyzer from SPECS (https://www. specs-group.com/nc/specs/productseries/detail/phoibos-150-ep/). The PEAXIS ultra-high-vacuum (UHV) components, mechanics and electronics were commercially built by PREVAC (https://www.prevac.eu/). A photograph of the PEAXIS spectrometer as installed in the BESSY II experimental hall is shown in Fig. 5(a), with the main components highlighted in color in the schematic drawing below in Fig. 5(b).

An important feature of PEAXIS is the possibility to rotate the RIXS spectrometer arm continuously around the sample for $2 \theta$ scattering angles between $33^{\circ}$ and $139^{\circ}$, providing a wavevector coverage of $0.058 \AA^{-1}$ to $0.190 \AA^{-1}$ at $200 \mathrm{eV}$ and $0.345 \AA^{-1}$ to $1.139 \AA^{-1}$ at $1200 \mathrm{eV}$ incident photon energy. 
(a)

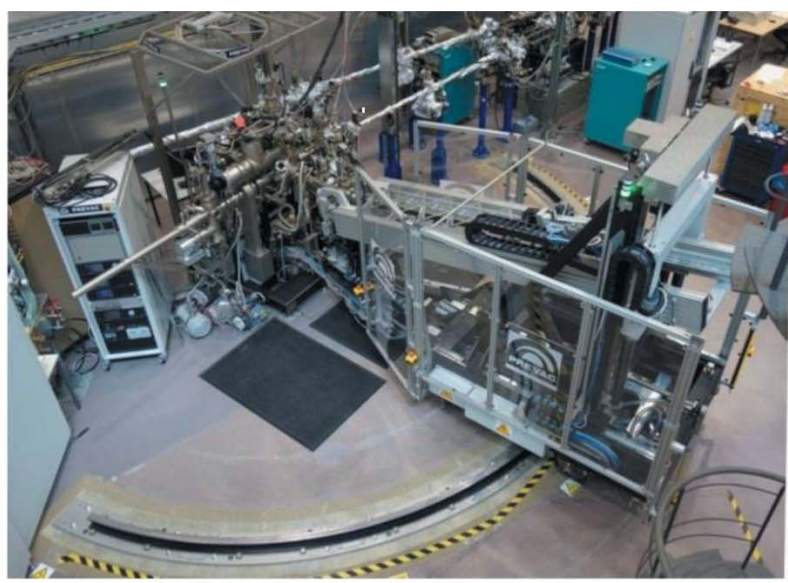

(b)

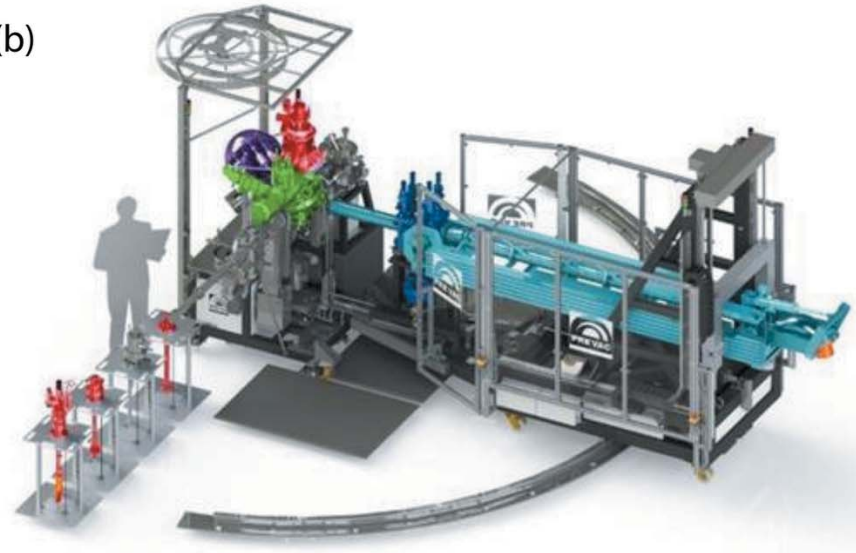

Figure 5

(a) Photograph of the PEAXIS station installed and in operation in the BESSY II experimental hall. (b) Schematic diagram of PEAXIS highlighting the main components: the rotatable arm of the RIXS spectrometer (light blue) together with the grating chamber (dark blue), the RIXS detector (orange), the sample chamber (green) with the available sample manipulators (red), the XPS detector (violet) and the load lock and sample preparation chamber (left side in front of the sample chamber).

The RIXS spectrometer operates with two spherical variableline-space (VLS) gratings for energy-resolving measurements in respective energy ranges of $200-600 \mathrm{eV}$ and $400-1200 \mathrm{eV}$. The complete RIXS design as well as an analytical calculation of the required parameters for the line densities of the VLS gratings and a numerical optimization of the overall parameters such as the total spectrometer arm length and considerations on optimum distances between sample position and analyzer grating at a given photon energy have been described previously (Lieutenant et al., 2016a,b). A more detailed description of the main components of PEAXIS is given in the following Subsections 3.1-3.3.

\subsection{Sample chamber and sample environments}

The sample chamber centered at the focal point of the beamline is preceeded by a load-lock chamber that is separately pumped to achieve UHV conditions prior to the transfer of the sample to the measurement position inside the sample chamber. The load-lock chamber hosts a sixfold sample holder that accommodates standard flag-style sample holders of $18 \mathrm{~mm} \times 21 \mathrm{~mm}$ size typically made out of tantalum or copper onto which the samples of optimal thickness $1.5 \mathrm{~mm}$ are attached by means of $\mathrm{Cu}$ tape or conductive Ag paint. Three out of the six positions on the load-lock sample holder can optionally be heated up to $800 \mathrm{~K}$, which may be used for thermal annealing purposes prior to the actual measurements. A mechanical cleaving tool allows for in situ cleaving of the samples at a pressure $<5 \times 10^{-9} \mathrm{mbar}$ if uncontaminated sample surfaces are required. Alternatively, the load-lock is equipped with an argon ion sputtering tool that can be used for a sputtering treatment of the sample surfaces.

A base stage located at the top of the sample chamber allows for sample positioning with four degrees of freedom, i.e. three orthogonal translations along the $x$ (along the photon beam), $y$ and $z$ directions and a rotation about an axis parallel to the $z$-direction $(\omega)$ as shown in Fig. 6. Three different sample manipulators can be inserted into this base stage for a different choice of samples (solid or liquid) and provide different sample temperature ranges. A closed-cycle helium (CCR) manipulator covers a temperature range from $10 \mathrm{~K}$ to $300 \mathrm{~K}$, while a second manipulator is available for the hightemperature range from $77 \mathrm{~K}$ up to $1000 \mathrm{~K}$. For both manipulators, sample temperatures below room temperature can be controlled with a precision of $0.1 \mathrm{~K}$. For temperatures above $300 \mathrm{~K}$, the sample temperature is derived from the heating power, which currently restricts the precision to $\pm 5 \mathrm{~K}$ below $350 \mathrm{~K}$ and above $700 \mathrm{~K}$ and to $\pm 10 \mathrm{~K}$ in the intermediate temperature range. An upgrade to a direct sample temperature measurement with higher precision over the whole temperature range $300-1000 \mathrm{~K}$ is planned for the near future. The two manipulators for solid samples provide two additional
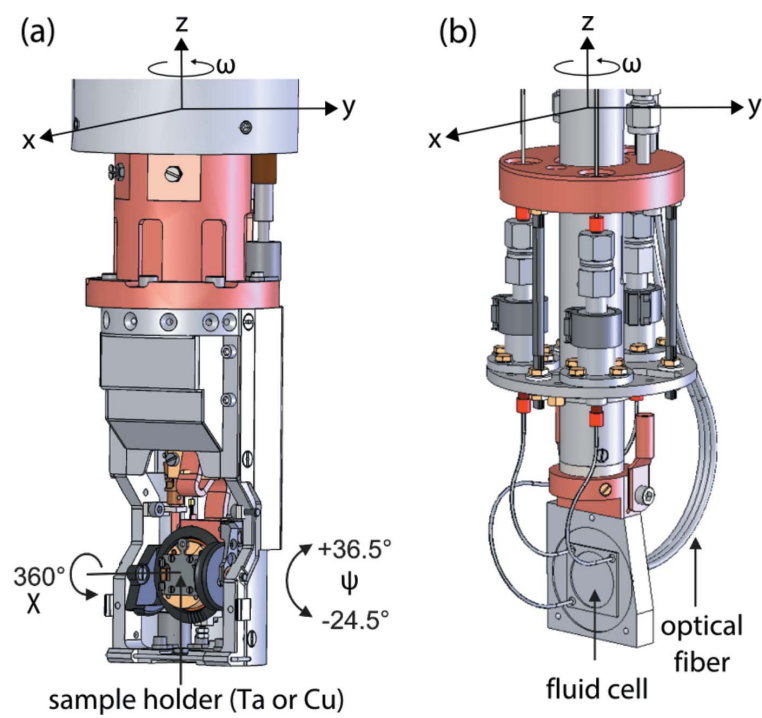

Figure 6

PEAXIS sample manipulators and motion degrees of freedom. (a) Solid sample manipulator, here shown for the low temperature range (CCR), and $(b)$ liquid sample manipulator. 
rotations [labeled $\chi$ and $\psi$ in Fig. 6(a)] around two orthogonal axes in the $x-y$ plane defined by the base stage. Hence, solid samples can be positioned by a total of six degrees of freedom. The third manipulator shown in Fig. 6(b) is designed for the measurement of liquids that are sealed in a fluid micro-cell with a thin membrane (about $100 \mathrm{~nm}$ thick) that allows for photon transmission. This liquid sample manipulator does not have any additional tilting/azimuthal rotation capabilities and allows the cell to be aligned with the four degrees of freedom of the base stage.

\subsection{RIXS spectrometer}

The RIXS spectrometer incorporates a $5 \mathrm{~m}$-long arm which can be rotated continuously about the sample position without breaking vacuum. A schematic drawing of the setup is shown in Fig. 7. The rotation or scattering angle $2 \theta$ defined as the angle between the incident photon beam and the emitted photons from the sample in the direction of the analyzer and detector can be varied between $33^{\circ}$ and $139^{\circ}$ with a precision of better than $0.03^{\circ}$. This angular range corresponds to accessible wavevectors $Q$ from $0.058 \AA^{-1}$ to $0.190 \AA^{-1}$ and $0.345 \AA^{-1}$ to $1.139 \AA^{-1}$ for photon energies of $200 \mathrm{eV}$ and $1200 \mathrm{eV}$, respectively. The angular range can be extended to $149^{\circ}$, provided the distance $r_{1}$ between the sample and the grating used to analyze the emitted photon energy is larger than $1071 \mathrm{~mm}$, which presently requires using incident photon energies between $550 \mathrm{eV}$ and $600 \mathrm{eV}$ and the low-energy grating.

The RIXS spectrometer is equipped with two spherically shaped VLS gratings. Both of them are mounted in the grating chamber on a grating manipulator. This manipulator has five motorized degrees of freedom that allow the selected grating to be positioned based on the calculated values for the chosen

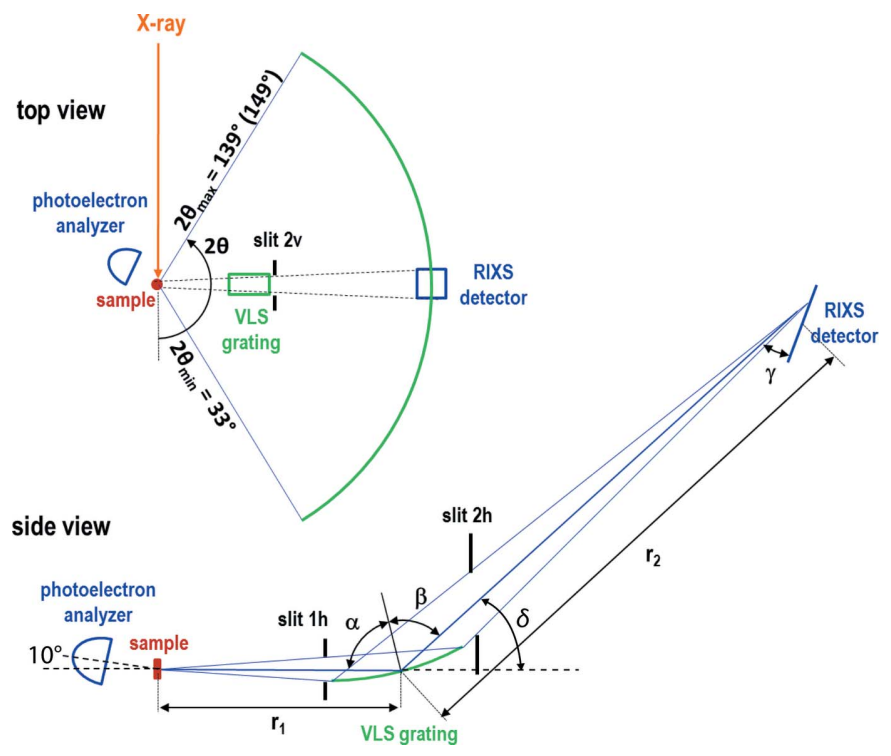

Figure 7

Schematic drawing of the RIXS spectrometer setup in the horizontal (top view, top panel) and in the vertical (side view, bottom panel) photon scattering planes, with angles and distances as discussed in the text. photon energy. The angle $\alpha$ between the photon beam emitted from the sample and the normal to the grating surface can be varied between $84^{\circ}$ and $90^{\circ}$ with a precision $\leq 10^{-5}$ degrees. A second rotation around the direction of the incident photon beam positions the grating such that its spherically shaped surface is symmetric with respect to the central photon beam. Three translations are necessary to align the grating with respect to height and distance to the sample and to switch between the two VLS gratings mounted on the grating holder.

The grating chamber (together with the grating manipulator) can be moved along the RIXS arm. By that movement, the distance between the sample and the center of the grating $\left(r_{1}\right)$ can be adjusted from $650 \mathrm{~mm}$ to $1250 \mathrm{~mm}$ with an accuracy of $0.01 \mathrm{~mm}$. To prevent any absorption of the soft X-rays in air, the area between the sample and the grating chambers is kept under vacuum within a bellow with a differential pumping chamber.

Both gratings are mounted with their gold-coated active surfaces facing upwards and thus the photons are dispersed in the vertical direction and detected at the distance $r_{2}$ by a cooled CCD detector (model iKon-L, cooled to $-100^{\circ}$ by a five-stage Peltier cooler to minimize the detector dark current to realize long exposure times) from Andor (https://andor. oxinst.com/). Similar to $r_{1}$, the distance $r_{2}$ between the grating and the detector can be adjusted to within $0.01 \mathrm{~mm}$. The available distance ranges between $2012 \mathrm{~mm}$ and $4080 \mathrm{~mm}$, with a tolerance of $0.01 \mathrm{~mm}$. The angle $\delta(c f$. Fig. 7) between the horizontal plane and the direction between the center of the grating and the center of the CCD detector can be varied between $0^{\circ}$ and $15^{\circ}$ with an accuracy of $0.001^{\circ}$.

The CCD chip has $2048 \times 2048$ channels and a pixel size of $13.5 \mu \mathrm{m} \times 13.5 \mu \mathrm{m}$ over its physical size of $27.6 \mathrm{~mm} \times$ $27.6 \mathrm{~mm}$. This detector size combined with the distances $r_{1}$ and $r_{2}$ of the RIXS arm allows RIXS spectra to be collected covering an energy range of $35 \mathrm{eV}$ at a typical incident photon energy of $\sim 530 \mathrm{eV}$ (O K-edge), or a range of $75 \mathrm{eV}$ at an incident energy of $\sim 930 \mathrm{eV}$ ( $\mathrm{Cu} L$-edge).

The inclination angle $\gamma$ between the surface of the CCD chip and the incoming photon beam can be varied between $15^{\circ}$ and $65^{\circ}$ with $0.001^{\circ}$ precision. By choosing a low inclination angle, the energy resolution of the CCD detector can be improved, because the number of pixels per angle and thus per energy is larger. However, for the smaller inclination angles, a portion of the lower detector channels is shadowed, which should be taken into consideration for experimental planning purposes. By choosing a large inclination angle, the energy range on the detector is increased at the cost of detector resolution.

In order to restrict the illuminated area on the active grating, three slits systems (one with vertical aperture, 'slit $2 \mathrm{v}$ ', and two with horizontal apertures, 'slit $1 \mathrm{~h}$ ' and 'slit $2 \mathrm{~h}$ ' in Fig. 7) are mounted within the grating chamber. These slit systems are also useful for reducing the number of photons that are scattered at the chamber walls and reach the CCD chip indirectly. They are therefore relevant for the suppression of photon background. The positions of these slit systems are shown in Fig. 7. 
Table 2

Overview of the two VLS gratings' parameters of PEAXIS.

Values in the upper part of the table correspond to the grating substrates provided by Zeiss GmbH (https://www.zeiss.com).

\begin{tabular}{|c|c|c|c|c|}
\hline Parameter & $\begin{array}{l}\text { Low energy } \\
\text { grating design }\end{array}$ & $\begin{array}{l}\text { Low energy } \\
\text { grating realization }\end{array}$ & $\begin{array}{l}\text { High energy } \\
\text { grating design }\end{array}$ & $\begin{array}{l}\text { High energy } \\
\text { grating realization }\end{array}$ \\
\hline Energy range $(\mathrm{eV})$ & $200-600$ & $200-600$ & $400-1200$ & $400-1200$ \\
\hline Radius (mm) & 27157 & 27071 & 41513 & 41345 \\
\hline Slope error tangential r.m.s. (arcsec) & $<0.1$ & 0.111 & $<0.1$ & 0.104 \\
\hline Slope error sagittal r.m.s. (arcsec) & $<2.5$ & 0.22 & $\leq 2.5$ & - \\
\hline Micro roughness r.m.s. (nm) & $\leq 0.3$ & $<0.19$ & $\leq 0.3$ & $<0.28$ \\
\hline Linear density $N_{0}\left(\right.$ lines $\left.\mathrm{mm}^{-1}\right)$ & 2400 & $2399.972 \pm 0.007$ & 2400 & $2399.400 \pm 0.005$ \\
\hline Linear coefficient $\left(\mathrm{mm}^{-2}\right)$ & $1.0942 \times 10^{-4}$ & $1.0940 \times 10^{-4} \pm 0.0005 \times 10^{-4}$ & $1.3058 \times 10^{-4}$ & $1.3081 \times 10^{-4} \pm 0.0003 \times 10^{-4}$ \\
\hline Quadratic coefficient $\left(\mathrm{mm}^{-3}\right)$ & $-9.37 \times 10^{-8}$ & $-9.36 \times 10^{-8} \pm 0.04 \times 10^{-8}$ & $-8.90 \times 10^{-8}$ & $-8.89 \times 10^{-8} \pm 0.02 \times 10^{-8}$ \\
\hline Cubic coefficient $\left(\mathrm{mm}^{-4}\right)$ & $1.70 \times 10^{-10}$ & $1.74 \times 10^{-10} \pm 0.07 \times 10^{-10}$ & $1.76 \times 10^{-10}$ & $1.79 \times 10^{-10} \pm 0.04 \times 10^{-10}$ \\
\hline Blaze angle $\left({ }^{\circ}\right)$ & 2.4 & $2.49 \pm 0.15(2 \sigma)$ & 1.9 & $2.13 \pm 0.19(2 \sigma)$ \\
\hline Apex angle $\left({ }^{\circ}\right)$ & $<174$ & $171.3 \pm 1.6(2 \sigma)$ & $<170$ & $172.9 \pm 0.9(2 \sigma)$ \\
\hline Micro roughness r.m.s. (nm) & $\leq 0.6$ & $0.27 \pm 0.05$ & $\leq 0.6$ & $0.32 \pm 0.13$ \\
\hline Au coating thickness $(\mathrm{nm})$ & 30 & $32 \pm 2$ & 30 & $31 \pm 3$ \\
\hline
\end{tabular}

The two VLS gratings used in the RIXS spectrometer were produced at Helmholtz-Zentrum Berlin, Germany (HZB). The required substrates were produced by the company Zeiss (https://www.zeiss.com). These spherically shaped blanks are made of silicon and have outer dimensions of $160 \mathrm{~mm} \times$ $40 \mathrm{~mm} \times 40 \mathrm{~mm}$ with an optical surface of $150 \mathrm{~mm} \times 15 \mathrm{~mm}$. These blanks were used to produce two VLS gratings each having an average line density of 2400 lines $\mathrm{mm}^{-1}$. The parameters of the low- and high-energy gratings have then been especially optimized for their respective photon energy ranges between $200-600 \mathrm{eV}$ and $400-1200 \mathrm{eV}$. The complete set of parameters of the two gratings including a comparison between targeted design values and experimentally verified parameters is given in Table 2.

The resulting reflection efficiencies of both gratings are shown in Fig. 8. They were measured at the reflectometer at

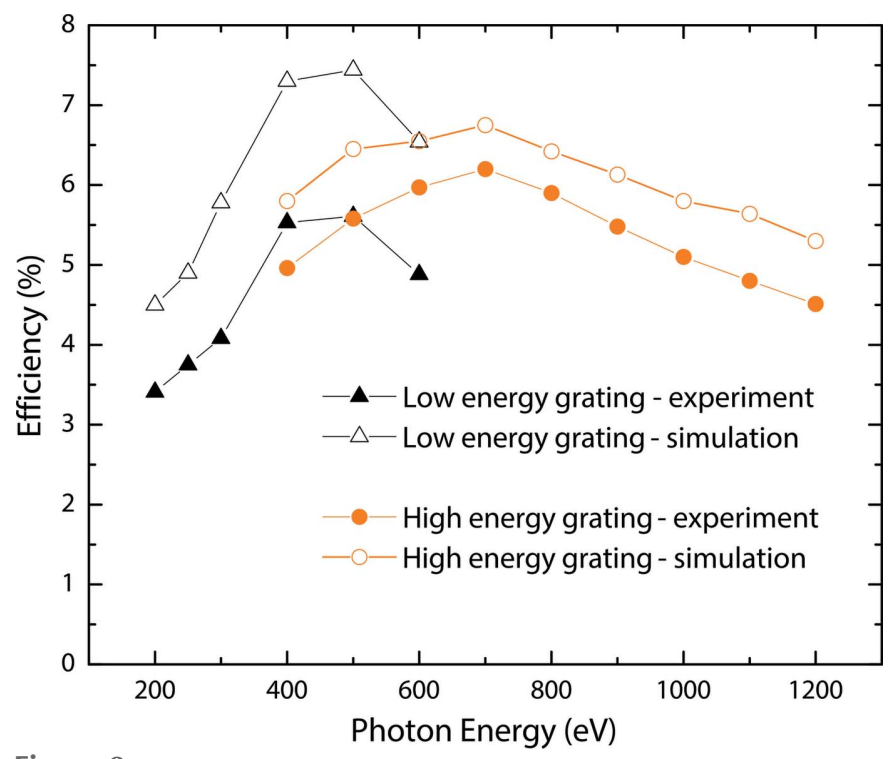

Figure 8

Measured (full symbols) and simulated (open symbols) reflection efficiencies of both VLS gratings currently employed at PEAXIS as a function of incident photon energy. the optics beamline at BESSY II (Schäfers \& Sokolov, 2016) and compared with simulations carried out with the raytracing software Ray-UI (Schäfers, 2008) which relies on a code developed by Neviere et al. (1982) to calculate reflection efficiencies of gratings. The overall realized reflection efficiencies for both gratings are comparable with generally achieved values of around $10 \%$, though the absolute values are systematically lower by about $10-20 \%$ than those predicted by the simulation. The reason for this discrepancy is currently being investigated. The general trend of the reflectivity curves as a function of incident photon energy, however, matches the simulation perfectly.

The combined energy resolution of the PEAXIS spectrometer over the accessible energy range $200-1200 \mathrm{eV}$ for the different operating modes of the beamline has been determined by measuring the energy width of the non-resonant elastic scattering signal from the polished surface of a $\mathrm{NiO}$ crystal positioned in the reflection condition. To maximize the intensity and shorten the measurement times, the RIXS spectrometer was operated in backscattering geometry $(2 \theta=$ $\left.135^{\circ}\right)$. The result of these measurements is shown in Fig. $9(a)$. The best resolution obtained on PEAXIS, $\sim 20 \mathrm{meV}$, is achieved for an incident photon energy of $\sim 200 \mathrm{eV}$ in the high-resolution mode of operation. For a typical incident energy of $\sim 530 \mathrm{eV}$ (O K-edge), the best energy resolution obtainable for reasonable photon flux is $\sim 50 \mathrm{meV}$, as shown in Table 2. This energy resolution is on a par with that realized at other recently built soft X-ray RIXS instruments at sources with similar brilliance conditions of photon flux. Fig. 9(b) shows an example for the profile of non-resonant elastically scattered photons, here at an incident photon energy of $500 \mathrm{eV}$ measured in high-flux mode using the low-energy grating. The experimental energy resolution function is well in agreement with a Gaussian lineshape.

The beamline and the RIXS spectrometer performance were simulated with the ray-tracing software $R A Y$-UI (Schäfers, 2008). The measured resolution is found to be in very good agreement with the simulation results with only slight deviations at the high-energy range limits of each spectro- 

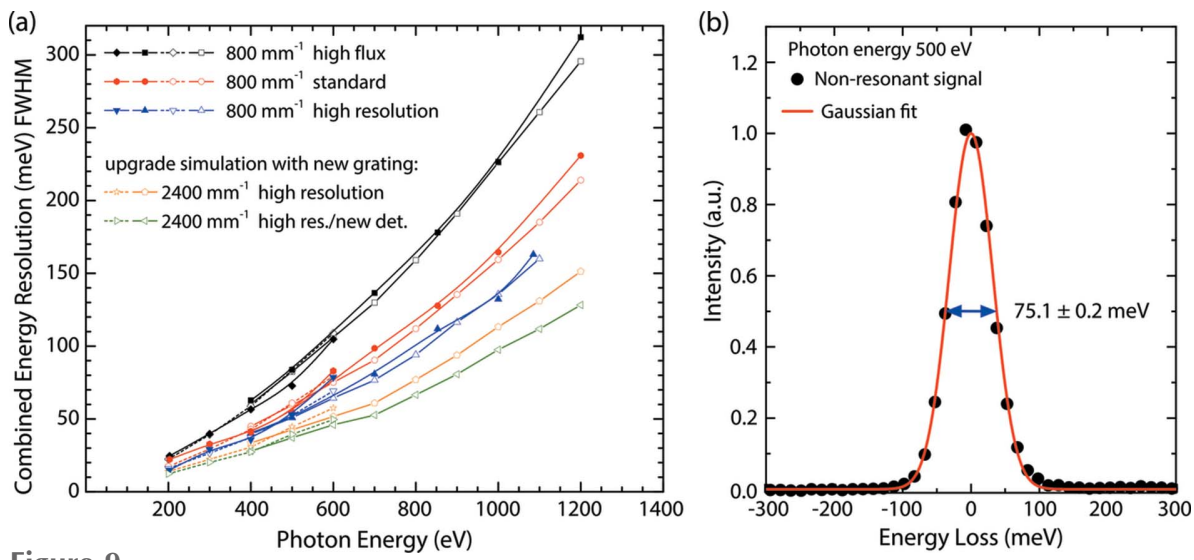

Figure 9

(a) Experimentally determined combined energy resolution of PEAXIS for low- and high-energy gratings (closed symbols) and comparison with simulated resolutions (open symbols). The simulated resolutions for planned upgrades including a new grating with increased line density and/ or a new detector with better spatial resolution are also shown. (b) Gaussian fit to non-resonant elastically scattered photons measured in high-flux mode.

meter grating. In addition to the simulations for the existing monochromator grating with 800 lines $\mathrm{mm}^{-1}$, Fig. 9(a) also shows simulation results for a foreseen upgraded monochromator grating with an increased line density of 2400 lines $\mathrm{mm}^{-1}$. These simulations were performed for the same parameters as for the 800 lines $\mathrm{mm}^{-1}$ grating [orange curve in Fig. $9(a)$ ] and for the same parameters in addition to a new detector upgraded to pixel sizes of $5 \mu \mathrm{m}$ in the energy axis [green curve in Fig. 9(a)]. After these planned grating and detector upgrades, a resolving power in excess of 10000 is envisioned for incident photon energies up to $1000 \mathrm{eV}$.

\subsection{Photoelectron spectrometer}

PEAXIS optionally allows for XPS studies within the same sample chamber. For these studies, photoelectrons which are emitted from the sample after the photon absorption can be collected by an electron analyzer (PHOIBOS 150 EP from SPECS) mounted at the sample chamber (see Figs. 5 and 7). In the standard position for XPS measurements with the RIXS arm located at $2 \theta=90^{\circ}$, the XPS detector is positioned at an angle of $55^{\circ}$ with respect to the incident X-ray beam in the horizontal and at an angle of $10^{\circ}$ in the vertical plane ( $c f$. bottom of Fig. 7). In addition to the possible rotation of the sample about the three manipulator axes $\omega, \psi$ and $\chi$, the XPS analyzer itself can be rotated about the beamline axis by an angle $\varphi$ (together with the sample chamber) in order to choose an optimal combination of the angles between the direction of incident photons and sample surface as well as between sample surface and the direction of the emitted electrons. Under normal operating conditions, the sample chamber is connected to the RIXS arm which restricts the angular range of the XPS detector to $-24.8^{\circ}<\varphi<10^{\circ}$. This range can be extended to $-28^{\circ}<\varphi<57^{\circ}$ upon disconnecting the RIXS arm from the sample chamber (rarely done in practice).

The XPS analyzer is equipped with a 2D CCD detector. It is therefore possible to perform angle-dependent measurements. An ultimate energy resolution of $\leq 2 \mathrm{meV}$ FWHM was confirmed as part of factory acceptance tests by measuring the $\mathrm{Xe} 5 p_{3 / 2}$ line at $12.130 \mathrm{eV}$ binding energy using the He-I excitation line.

Being a commercial instrument, the PHOIBOS 150 EP analyzer can be used in different modes of operation. For XPS measurements, the 'fixed analyzer transmission' (FAT) mode is typically used, in which the excitation and the pass energies of the analyzer are kept constant whereas the kinetic energy of the electrons is varied. The energy resolution of the analyzer is constant throughout the scan. A typical measurement in this mode to illustrate the XPS capabilities of PEAXIS is shown in Fig. 10 for a $100 \mathrm{~nm}$ Au film (Sigma Aldrich).

The figure shows a survey scan over a wide energy range $(a)$ and a fine scan at the $\mathrm{Au} 4 f$ lines $(b)$ with the typical energy resolution of $0.43 \mathrm{eV}$ FWHM of the measurement, which was determined by fitting the data using the CasaXPS software suite (Walton et al., 2010). For this set of measurements, the beamline was operated in standard mode at an incident photon energy of $1000 \mathrm{eV}$, with the PHOIBOS $150 \mathrm{EP}$ set to medium area mode with entrance slit $0.2 \mathrm{~mm} \times 20 \mathrm{~mm}$, open exit slit and a pass energy of $E_{\mathrm{p}}=5 \mathrm{eV}$.
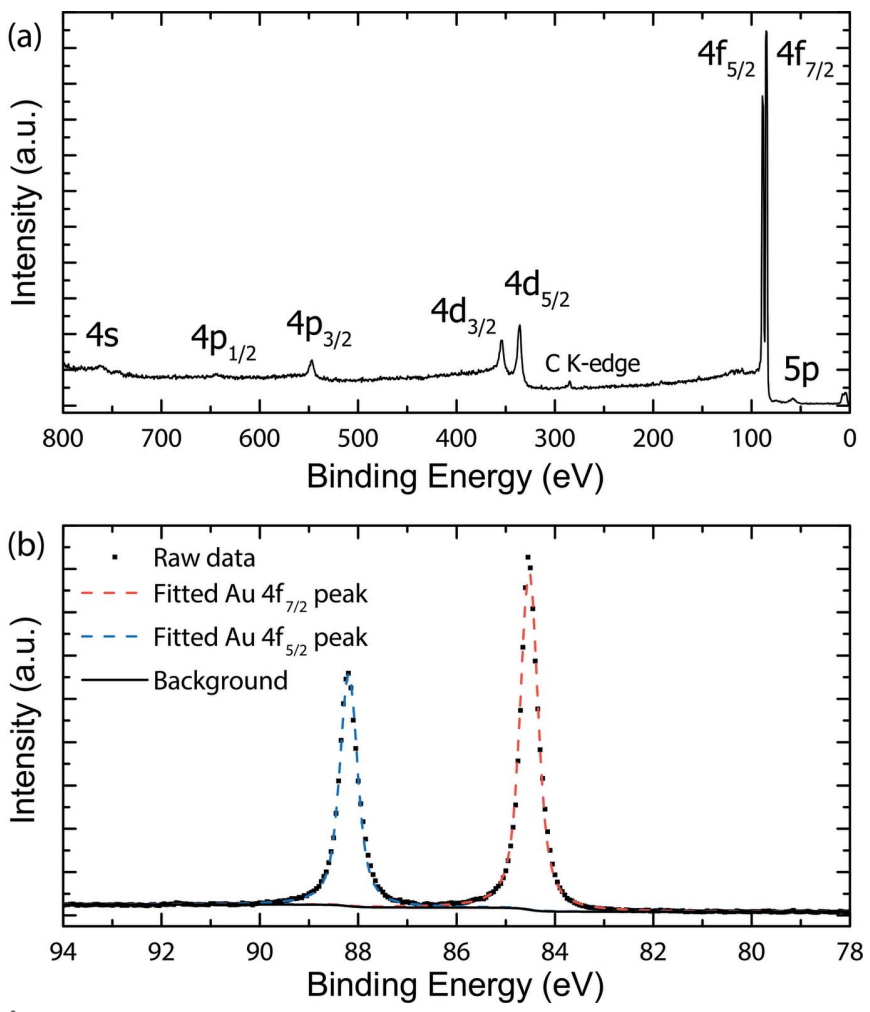

Figure 10

Illustration of XPS capabilities of PEAXIS on a $100 \mathrm{~nm}$-thick Au film. A survey scan $(a)$ and a fine scan at the Au $4 f$ lines $(b)$ recorded for $1000 \mathrm{eV}$ incident photon energy and standard beamline mode. 
In addition to the FAT mode, angle-depended XPS measurements can be performed with three different angular dispersion modes. For occupied-state measurements, the analyzer is typically set to the 'constant final state' mode, for which the kinetic energy of the electrons and the pass energy are kept constant while varying the excitation energy. The 'constant initial state' mode is correspondingly used for empty-state spectroscopy measurements, in which the excitation energy and the measured kinetic energy of the electrons are varied simultaneously and with equal step size. Finally, Auger electrons are typically collected in the 'fixed retarding ratio' mode. In this mode, the excitation energy is kept constant, but the measured kinetic and pass energies are varied to keep the ratio between the retarding potential and the pass energy constant.

\section{Scientific examples}

To illustrate the instrumental capabilities and the performance of PEAXIS, two samples - one solid and one liquid - have been studied by RIXS.

\subsection{Excitations in single-crystalline $\mathrm{NiO}$}

RIXS is a coherent photon-in/photon-out process and transitions from initial to final states are not subject to the dipole selection rules as they are in other optical spectroscopies. The technique is particularly suited to probe $d-d$ excitations and charge-transfer excitations in $3 d$ or $4 d$ transition metal complexes. In nickel oxide (NiO), these excitations are intimately related to the sixfold oxygen coordination of each $\mathrm{Ni}$ atom. Six oxygen ligands on a regular octahedron cause a splitting of Ni $3 d$ states by symmetry, combined with a $\mathrm{Ni}-3 d+\mathrm{O}-2 p$ hybridization.

To probe the electronic $\mathrm{Ni} 3 d$ states in $\mathrm{NiO}$, incident photon energies were chosen close to the $\mathrm{Ni} L_{3}$ resonance around $853 \mathrm{eV}$. For that purpose, total electron yield (TEY) measurements were first performed to accurately determine the $L_{3}$ resonance energy as shown in Fig. 11.

As the emitted RIXS spectrum depends sensitively on the incident photon energy [see Fig. 12(b)], the precise resonance energy for the $L_{3}$ (and $L_{2}$, or any absorption edge of interest) excitation should be determined by TEY upon X-ray absorption before the collection of RIXS spectra. Fig. 12

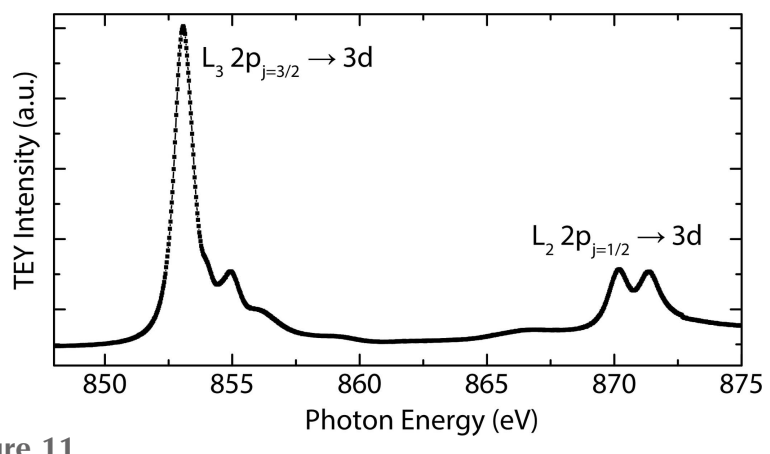

Figure 11

TEY spectrum of $\mathrm{NiO}$ around the $\mathrm{Ni} L_{3}$ - and $L_{2}$-edges.
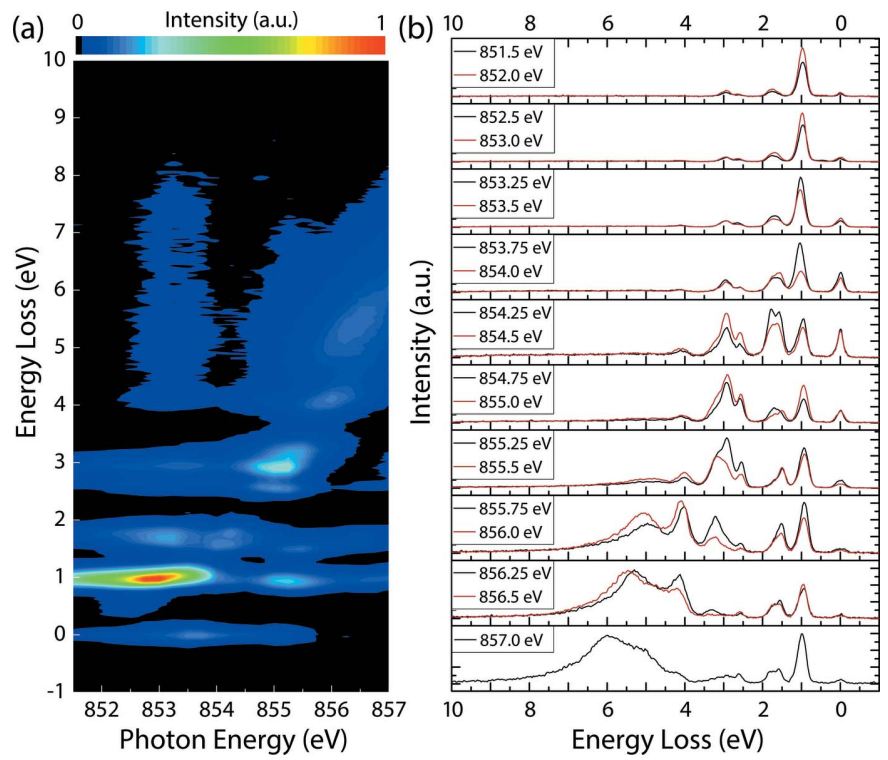

Figure 12

(a) Two-dimensional RIXS map of NiO displaying regions with high intensity corresponding to the $d-d$ excitations at constant energy transfers and charge transfer excitations for energy transfers continuously increasing with increasing incident photon energy. (b) The raw RIXS spectra collected to create the RIXS map.

shows experimental data measured at PEAXIS which are in good agreement with experimental and calculated data by Betto et al. (2017) and with earlier data by Ghiringhelli et al. (2005).

The RIXS spectra shown in Fig. 12(b) were collected in the standard mode of the beamline yielding an energy resolution of $136 \mathrm{meV}$ with incident photon energies from $851.5 \mathrm{eV}$ well below the $L_{3}$ absorption edge to $857.0 \mathrm{eV}$ well beyond the $L_{3}$ edge. To avoid strong elastic scattering from the sample, the scattering angle of the RIXS spectrometer was set to $110^{\circ}$ close to the linear polarization direction of the incident X-ray beam. In order to even further suppress the elastic scattering, the sample was rotated by an angle of $4^{\circ}$ away from the elastic reflection condition at $\theta=59^{\circ}$. The data acquisition for each of the spectra shown in Fig. 12(b) took 15 minutes.

For incident photon energies below the $L_{3}$ resonance of $853 \mathrm{eV}$, there is very little variation in the measured spectra. Above the resonance, new features become more pronounced with increasing photon energy. One part of these spectra is related to the $d-d$ excitations (up to about $4 \mathrm{eV}$ ), which can be described by the crystal field model of $\mathrm{NiO}$ (Uldry et al., 2012). The second, dispersing, part at higher energy transfers is related to the charge transfer excitations and can be described by the Anderson impurity model (Ghiringhelli et al., 2005; Matsubara et al., 2005; Magnuson et al., 2002).

The main peak at $1 \mathrm{eV}$ (which is present in all measured spectra) can be interpreted as corresponding to the crystal field splitting parameter $10 \mathrm{Dq}$, which is given as the energy splitting between the singly occupied $e_{\mathrm{g}}\left(x^{2}-y^{2}, z^{2}\right)$ and doubly occupied $t_{2 g}(x y, y z, z x)$ orbitals in the crystal field model. All peaks between $1 \mathrm{eV}$ and $4 \mathrm{eV}$ are caused by a redistribution of the $3 d$ electrons among the available orbitals 
during the scattering process (localized at the $\mathrm{Ni}$ ion) from an initial $3 d^{8}$ ground state to a new configuration $3 d^{8 *}$ at higher energy with respect to the ground state, effectively leading to an excited $d$-state, thus called $d-d$ excitation. This energy difference is detected in the RIXS measurement as an energy loss in the scattering process. If the incident photon energy is high enough to populate also the configuration with a ligand hole $\left(3 d^{9} \underline{L}\right)$, then a charge transfer excitation with a larger energy loss can occur. The energy difference between these two configurations $\left(3 d^{9} \underline{L}, 3 d^{8}\right)$ is called the charge transfer energy $\Delta=E\left(3 d^{9} \underline{L}\right)-E\left(3 d^{8}\right)$. A more detailed analysis has been given by Ghiringhelli et al. (2005) and references therein. For a comprehensive overview of all features and their intensity as a function of the incident photon energy, the individual RIXS spectra can be combined into a two-dimensional RIXS map, shown in Fig. 12(a).

In addition to probing electronic excitations, RIXS allows magnetic excitations to be probed in single-crystalline samples (Betto et al., 2017; Ament et al., 2009; de Groot et al., 1998). The mechanism by which the magnetic excitations are created depends, however, on the particular RIXS process. Spin and orbital degrees of freedom couple if the intermediate state, for example at an $L$-edge, involves a core-hole with strong spinorbit coupling. In a direct process, the angular momentum of photons can then be transferred to the spins to create a magnetic excitation (spin flip excitation) (de Groot et al., 1998; Ament, 2010). If the RIXS process involves a core-hole without spin-orbit coupling, such as for example at a $K$-edge, the transient intermediate state is a local, magnetic impurity that perturbs the system and causes a magnetic excitation (Ament, 2010). This indirect RIXS channel exists in principle also for spin-orbit coupled core-holes but has been shown to contribute only in higher order to the spectral weight.

Probing low-energy magnetic excitations is challenging, as their energies are typically one or two orders of magnitude smaller than those of electronic $d-d$ excitations. The success of the measurements therefore depends on the energy resolution the spectrometer can provide at the relevant resonance edges. Fig. 13 shows a one-magnon and a two-magnon excitation in $\mathrm{NiO}(001)$ measured with an energy resolution of $130 \mathrm{meV}$ in the standard mode of operation at a sample temperature of $T=20 \mathrm{~K}$. This RIXS measurement was performed at the Ni $L_{3}$-edge to maximize the spectral weight of the one-magnon excitation. As previously discussed, a scattering angle of $2 \theta=$ $110^{\circ}$ and an angle $\theta=59^{\circ}$ was selected to reduce the elastic scattering from the sample. At this angular setting, the wavevector transfer $Q$ is $\sim 0.7 \AA^{-1}$, which corresponds to the Brillouin zone boundary along the [110] direction and which thus allows to observe the maximum of the magnon dispersion at $\sim 110 \mathrm{meV}$ (Hutchings \& Samuelsen, 1972). The beamline operated in standard mode. The data accumulation time per spectrum was $60 \mathrm{~min}$. The one-magnon peak was fitted at $97 \mathrm{meV} \pm 7 \mathrm{meV}$ with a FWHM of $141 \mathrm{meV} \pm 6 \mathrm{meV}$ and the two-magnon peak at $228 \mathrm{meV} \pm 17 \mathrm{meV}$ with FWHM of $160 \mathrm{meV} \pm 30 \mathrm{meV}$.

Our results demonstrate the feasibility to study magnetic excitations with RIXS at PEAXIS. They are in good agree-

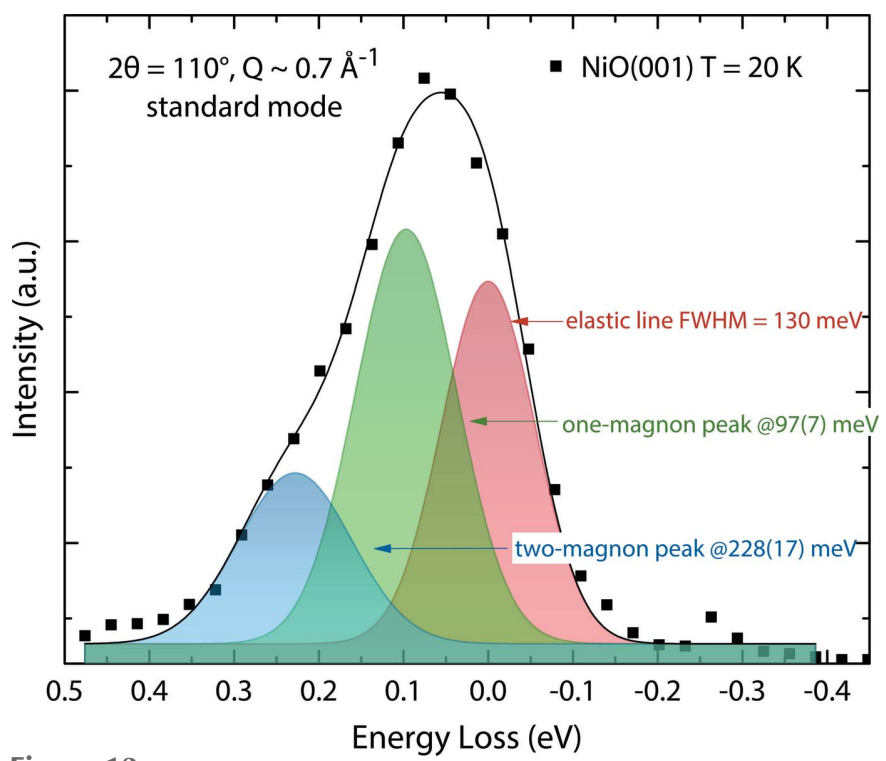

Figure 13

One- and two-magnon excitation in $\mathrm{NiO}$ close to the elastic signal.

ment with recent results from Betto et al. (2017) who performed magnon dispersion measurements at the ID32 beamline at the ESRF with a resolving power that was by a factor of three better than that presently achievable at PEAXIS, corresponding to an energy resolution of $40 \mathrm{meV}$ at that beamline. Betto et al. (2017) observed the one-magnon peak at around $100 \mathrm{meV}$ for $Q=0.7 \AA^{-1}$ with a FWHM width of $50-60 \mathrm{meV}$ and the two-magnon peak at $190 \mathrm{meV}$ with a FWHM of $\sim 150 \mathrm{meV}$. The peak positions extracted from our measurements agree well with these latter results.

\subsection{Vibrational excitations in liquid acetone}

Using RIXS, vibrational modes in individual molecules in the liquid state (Rubensson et al., 2013; Schreck et al., 2016) and crystals (Ament et al., 2011; Lee et al., 2013) can be investigated. This particular scattering channel is a direct consequence of the coupling between the electronic charge distribution in the intermediate state and the nuclear dynamics (Ament et al., 2011), based on the idea that the locally perturbed charge distribution in the transient intermediate state displaces the neighboring ions from their equilibrium positions and therefore causes molecular or lattice vibrations (van Veenendaal, 2015).

Here, RIXS data from liquid acetone are shown in Fig. 14. The presented spectra illustrate the capability of PEAXIS to study vibrational excitations in soft or liquid matter samples. For these measurements, a static fluid cell with a $75 \mathrm{~nm}$ thin entrance window made of $\mathrm{Si}_{3} \mathrm{~N}_{4}$ of $0.5 \mathrm{~mm} \times 0.5 \mathrm{~mm}$ size was used to measure liquid acetone (volume of $5 \mu \mathrm{l}$ ) in the ultrahigh vacuum of the sample chamber. The measurements were carried out in the standard mode of operation of the beamline to have sufficient flux to compensate for the X-ray absorption in the entrance window of the cell. The incident energy was set to $531 \mathrm{eV}$ (O $K$-edge) to excite a $1 s$ electron into a $\pi^{*}$ antibonding orbital of acetone (Schreck et al., 2016). The resulting 


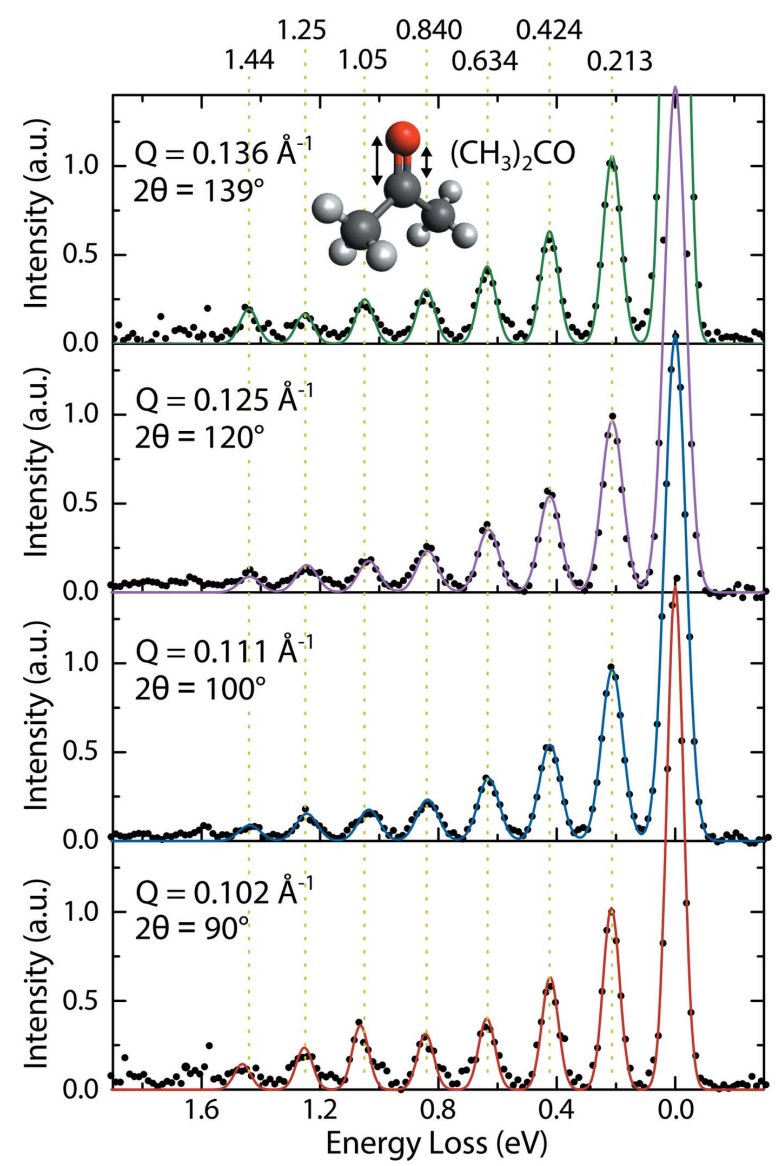

Figure 14

The vibrational excitations in liquid acetone were measured at the $\mathrm{O} K$ edge of $531 \mathrm{eV}$ with an energy resolution of $63 \mathrm{meV}$. The intensity is normalized to the first vibrational peak.

energy resolution at that incident photon energy was $\sim 63 \mathrm{meV}$, which is sufficient to resolve the $\mathrm{C}=\mathrm{O}$ stretching mode of liquid acetone at a fundamental energy of $\sim 210 \mathrm{meV}$ (Schreck et al., 2016). To investigate whether the wavevector $Q$ has an influence on the vibrational progression, RIXS spectra at four different scattering angles $\left(2 \theta=90^{\circ}, 100^{\circ}, 120^{\circ}\right.$ and $139^{\circ}$ ) have been recorded. With the current signal-tonoise ratio of PEAXIS, seven peaks at the fundamental frequency and its overtones can be confidently identified (see Fig. 14). These could be satisfactorily described by resolutionlimited Gaussian lineshapes, resulting in the following peak positions: $213 \pm 2 \mathrm{meV}, 424 \pm 2 \mathrm{meV}, 634 \pm 3 \mathrm{meV}, 840 \pm$ $3 \mathrm{meV}, 1048 \pm 12 \mathrm{meV}, 1248 \pm 6 \mathrm{meV}$ and $1445 \pm 12 \mathrm{meV}$, in excellent agreement with previously reported results by Schreck et al. (2016). The four spectra do not show any dependence on scattering angle and thus no noticeable wavevector dependence in the small $Q$-region probed by the RIXS measurements in this setup.

\section{Conclusion and outlook}

PEAXIS is a versatile X-ray spectrometer offering $Q$-dependent RIXS measurements on solid and liquid samples over a wide temperature range. The performance of the beamline and the RIXS spectrometer were demonstrated by measurements on $\mathrm{N}_{2}$ and $\mathrm{Ne}$ gas as well as by measurements of typical excitations. Charge transfer excitations and $d-d$ excitations were investigated at the $L_{3}$-edge of $\mathrm{Ni}$ within a high-quality $\mathrm{NiO}$ crystal. The same crystal was used to measure single and double magnon excitations. To demonstrate the capability to measure liquid samples, the vibrational excitations in liquid acetone were also presented. The results from these measurements clearly show that PEAXIS is very well suited for the study of electronic excitations and, limited by the energy resolution, also for the study of quasi-particle excitations. On PEAXIS, magnetic and phonon excitations at $\sim 100 \mathrm{meV}$ can be resolved at typical transition metal $L$-edges energies. To improve the energy resolution of the beamline, its monochromator will be upgraded by an additional grating of higher line density [2400 lines $\mathrm{mm}^{-1}, c f$. Fig. $\left.9(a)\right]$. After this upgrade, the combined energy resolution of the beamline and the RIXS spectrometer in high-resolution mode will be increased from currently $94 \mathrm{meV}(136 \mathrm{meV})$ to $77 \mathrm{meV}$ $(113 \mathrm{meV})$ at a photon energy of $800 \mathrm{eV}(1000 \mathrm{eV})$. Matching the beamline resolution with the resolution of the spectrometer, a new detector is envisioned with $5 \mu \mathrm{m}$ pixel size which will allow for an additional increase of the resolution to $67 \mathrm{meV}$ at $800 \mathrm{eV}$ and $98 \mathrm{meV}$ at $100 \mathrm{eV}$. Furthermore, a replacement of the undulator at the beamline is foreseen. The new undulator will provide a higher photon flux, especially at photon energies above $1200 \mathrm{eV}$ and, in addition, an adjustable photon beam polarization to tailor to the experimental requirements. An upgrade of the RIXS CCD detector to a model with smaller pixel size will further improve the energy resolution, targeting a resolving power of $\geq 10000$ at $1000 \mathrm{eV}$.

\section{Acknowledgements}

We would like to thank Peter Guttmann and Matthias Mast for their great work building up and commissioning the main part of the U41-PEAXIS beamline. The help of the teams from the HZB Department Optics and Beamlines, the Department Precision Gratings, the Department Nanometre Optics and Technology and the Department Scientific Technical Infrastructure is gratefully acknowledged. We would like to thank Katharina Fritsch for helpful discussions and suggestions to improve the manuscript as well as invaluabe help with the figures. The project was funded in part by the German BMBF under Förderkennzeichen 05K13KE4.

\section{References}

Ament, L. J. P. (2010). Resonant inelastic X-ray scattering studies of elementary excitations. PhD thesis, Leiden University, The Netherlands.

Ament, L. J. P., Ghiringhelli, G., Sala, M. M., Braicovich, L. \& van den Brink, J. (2009). Phys. Rev. Lett. 103, 117003.

Ament, L. J. P., van Veenendaal, M. \& van den Brink, J. (2011). Europhys. Lett. 95, 27008.

Betto, D., Peng, Y. Y., Porter, S. B., Berti, G., Calloni, A., Ghiringhelli, G. \& Brookes, N. B. (2017). Phys. Rev. B, 96, 020409. 
Brookes, N., Yakhou-Harris, F., Kummer, K., Fondacaro, A., Cezar, J., Betto, D., Velez-Fort, E., Amorese, A., Ghiringhelli, G., Braicovich, L., Barrett, R., Berruyer, G., Cianciosi, F., Eybert, L., Marion, P., van der Linden, P. \& Zhang, L. (2018). Nucl. Instrum. Methods Phys. Res. A, 903, 175-192.

Brzhezinskaya, M. (2013). Ray-tracing results for construction of the 2nd branch of the METRIX-TXM-beamline. Internal HZB Report. Helmholtz-Zentrum Berlin für Materialien und Energie, Berlin, Germany.

Chiuzbăian, S. G., Hague, C. F., Avila, A., Delaunay, R., Jaouen, N., Sacchi, M., Polack, F., Thomasset, M., Lagarde, B., Nicolaou, A., Brignolo, S., Baumier, C., Lüning, J. \& Mariot, J.-M. (2014). Rev. Sci. Instrum. 85, 043108.

Chuang, Y.-D., Shao, Y.-C., Cruz, A., Hanzel, K., Brown, A., Frano, A., Qiao, R., Smith, B., Domning, E., Huang, S.-W., Wray, L. A., Lee, W.-S., Shen, Z.-X., Devereaux, T. P., Chiou, J.-W., Pong, W.-F., Yashchuk, V. V., Gullikson, E., Reininger, R., Yang, W., Guo, J., Duarte, R. \& Hussain, Z. (2017). Rev. Sci. Instrum. 88, 013110.

Couto, R. C., Cruz, V. V., Ertan, E., Eckert, S., Fondell, M., Dantz, M., Kennedy, B., Schmitt, T., Pietzsch, A., Guimarães, F. F., Agren, H., Gel'mukhanov, F., Odelius, M., Kimberg, V. \& Föhlisch, A. (2017). Nat. Commun. 8, 14165.

Feifel, R., Andersson, M., Öhrwall, G., Sorensen, S., Piancastelli, M., Tchaplyguine, M., Björneholm, O., Karlsson, L. \& Svensson, S. (2004). Chem. Phys. Lett. 383, 222-229.

Ghiringhelli, G., Matsubara, M., Dallera, C., Fracassi, F., Gusmeroli, R., Piazzalunga, A., Tagliaferri, A., Brookes, N. B., Kotani, A. \& Braicovich, L. (2005). J. Phys. Condens. Matter, 17, 5397-5412.

Groot, F. M. F. de, Kuiper, P. \& Sawatzky, G. A. (1998). Phys. Rev. B, 57, 14584-14587.

Hepting, M., Chaix, L., Huang, E. W., Fumagalli, R., Peng, Y. Y., Moritz, B., Kummer, K., Brookes, N. B., Lee, W. C., Hashimoto, M., Sarkar, T., He, J.-F., Rotundu, C. R., Lee, Y. S., Greene, R. L., Braicovich, L., Ghiringhelli, G., Shen, Z. X., Devereaux, T. P. \& Lee, W. S. (2018). Nature, 563, 374-378.

Huang, D. J. \& Chen, C. T. (2018). Synchrotron Radiat. News, 31(2), 3-6.

Hutchings, M. T. \& Samuelsen, E. J. (1972). Phys. Rev. B, 6, 34473461.

Jarrige, I., Bisogni, V., Zhu, Y., Leonhardt, W. \& Dvorak, J. (2018). Synchrotron Radiat. News, 31(2), 7-13.

Kato, M., Morishita, Y., Oura, M., Yamaoka, H., Tamenori, Y., Okada, K., Matsudo, T., Gejo, T., Suzuki, I. \& Saito, N. (2007a). J. Electron Spectrosc. Relat. Phenom. 160, 39-48.

Kato, M., Morishita, Y., Oura, M., Yamaoka, H., Tamenori, Y., Okada, K., Matsudo, T., Gejo, T., Suzuki, I. H. \& Saito, N. (2007b). AIP Conf. Proc. 879, 1121-1124.
Lee, W. S., Johnston, S., Moritz, B., Lee, J., Yi, M., Zhou, K. J., Schmitt, T., Patthey, L., Strocov, V., Kudo, K., Koike, Y., van den Brink, J., Devereaux, T. P. \& Shen, Z. X. (2013). Phys. Rev. Lett. 110, 265502.

Lieutenant, K., Hofmann, T., Schulz, C., Yablonskikh, M. V., Habicht, K. \& Aziz, E. F. (2016a). J. Electron Spectrosc. Relat. Phenom. 210, $54-65$.

Lieutenant, K., Hofmann, T., Zendler, C., Schulz, C., Aziz, E. \& Habicht, K. (2016b). J. Phys. Conf. Ser. 738, 012104.

Magnuson, M., Butorin, S. M., Agui, A. \& Nordgren, J. (2002). J. Phys. Condens. Matter, 14, 3669-3676.

Martin, T. \& Koch, A. (2006). J. Synchrotron Rad. 13, 180-194.

Matsubara, M., Uozumi, T., Kotani, A. \& Claude Parlebas, J. (2005). J. Phys. Soc. Jpn, 74, 2052-2060.

Meyers, D., Nakatsukasa, K., Mu, S., Hao, L., Yang, J., Cao, Y., Fabbris, G., Miao, H., Pelliciari, J., McNally, D., Dantz, M., Paris, E., Karapetrova, E., Choi, Y., Haskel, D., Shafer, P., Arenholz, E., Schmitt, T., Berlijn, T., Johnston, S., Liu, J. \& Dean, M. P. M. (2018). Phys. Rev. Lett. 121, 236802.

Neviere, M., Flamand, J. \& Lerner, J. (1982). Nucl. Instrum. Methods Phys. Res. 195, 183-189.

Pietzsch, A., Sokolov, A., Blume, T., Neppl, S., Senf, F., Siewert, F. \& Föhlisch, A. (2018). Synchrotron Radiat. News, 31(2), 20-25.

Rubensson, J.-E., Hennies, F. \& Pietzsch, A. (2013). J. Electron Spectrosc. Relat. Phenom. 188, 79-83.

Schäfers, F. (2008). Modern Developments in X-ray and Neutron Optics, ch. 2, pp. 9-41. Springer Series in Optical Sciences.

Schäfers, F. \& Sokolov, A. (2016). J. Large-Scale Res. Facil. 2, A50.

Schlappa, J., Kumar, U., Zhou, K. J., Singh, S., Mourigal, M., Strocov, V. N., Revcolevschi, A., Patthey, L., Rønnow, H. M., Johnston, S. \& Schmitt, T. (2018). Nat. Commun. 9, 5394.

Schreck, S., Pietzsch, A., Kennedy, B., Såthe, C., Miedema, P. S., Techert, S., Strocov, V. N., Schmitt, T., Hennies, F., Rubensson, J.-E. \& Föhlisch, A. (2016). Sci. Rep. 6, 20054.

Strocov, V. N., Schmitt, T., Flechsig, U., Schmidt, T., Imhof, A., Chen, Q., Raabe, J., Betemps, R., Zimoch, D., Krempasky, J., Wang, X., Grioni, M., Piazzalunga, A. \& Patthey, L. (2010). J. Synchrotron Rad. 17, 631-643.

Uldry, A., Vernay, F. \& Delley, B. (2012). Phys. Rev. B, 85, 125133.

Vaz da Cruz, V., Gel'mukhanov, F., Eckert, S., Iannuzzi, M., Ertan, E., Pietzsch, A., Couto, R. C., Niskanen, J., Fondell, M., Dantz, M., Schmitt, T., Lu, X., McNally, D., Jay, R. M., Kimberg, V., Föhlisch, A. \& Odelius, M. (2019). Nat. Commun. 10, 1013.

Veenendaal, M. van (2015). Theory of Inelastic Scattering and Absorption of X-rays, p. 221. Cambridge University Press.

Walton, J., Wincott, P., Fairley, N. \& Carrick, A. (2010). Peak Fitting with Casa XPS: A Casa Pocket Book, v.2.3.2. Acolyte Science, Knutsford, Cheshire, UK. 